Cahiers $d u$ MONDE RUSSE

\section{Cahiers du monde russe}

Russie - Empire russe - Union soviétique et États indépendants

50/2-3 | 2009

L'Europe orientale, 1650-1730. Crises, conflits et renouveau

\title{
The Politics of Knowledge in Central AsiaSarah
}

AMSLER

, Londres - New York : Routledge, 2007, vii + 188 p., bibliographie, index

\section{Stéphane A. Dudoignon}

\section{(2) OpenEdition}

\section{Journals}

Édition électronique

URL : https://journals.openedition.org/monderusse/9799

DOI : 10.4000/monderusse. 9799

ISSN : $1777-5388$

Éditeur

Éditions de l'EHESS

Édition imprimée

Date de publication : 15 septembre 2009

ISBN : 978-2-7132-2260-3

ISSN : $1252-6576$

Référence électronique

Stéphane A. Dudoignon, "The Politics of Knowledge in Central AsiaSarah AMSLER », Cahiers du monde russe [En ligne], 50/2-3 | 2009, mis en ligne le 14 janvier 2013, consulté le 03 septembre 2022 URL : http://journals.openedition.org/monderusse/9799; DOl : https://doi.org/10.4000/monderusse. 9799

Ce document a été généré automatiquement le 3 septembre 2022

Tous droits réservés 


\section{The Politics of Knowledge in Central AsiaSarah AMSLER}

, Londres - New York : Routledge, 2007, vii + 188 p., bibliographie, index

Stéphane A. Dudoignon

\section{Sarah AMSLER, The Politics of Knowledge in Central Asia, Science between Marx and the Market, Londres - New York : Routledge, 2007, vii + 188 p., bibliographie, index}

1 Avec l'ambition de construire une représentation globale de la sociologie et de la politique du savoir en Asie centrale, Sarah Amsler s'est concentrée sur le «travail de frontière " produit par les chercheurs du Kirghizstan pour définir le champ de la sociologie, en regard du politique en particulier, pendant les dernières années de la période soviétique (1985-1991) et la première décennie d'indépendance du pays (jusqu'en 2001). Le concept sociologique de boundary work est utilisé pour étudier la manière selon laquelle des domaines de connaissance sont construits, maintenus et transformés, à la fois au sein même des communautés scientifiques et vis-à-vis de la sphère publique ${ }^{82}$. Si ce dernier auteur se fonde assez classiquement (depuis Thomas S. Kuhn) sur le postulat que les disciplines scientifiques sont socialement et institutionnellement construites, il porte toutefois un intérêt tout particulier à l'évolution de la frontière entre les sciences, leur logique propre, celle de la recherche, et d'autres champs, le champ politique tout spécialement.

L'un des impacts les plus immédiats de la mise en œuvre de cette méthode sur les réalités centrasiatiques est la remise en cause du concept de «transition » postsoviétique dans la vie culturelle et intellectuelle de l'Asie centrale, voire une déconstruction pure et simple du concept de progrès intellectuel, qui fut à la base de nombreuses réformes et réajustements académiques des années 1990 et continue de constituer la référence ultime de nombreux protagonistes locaux des sciences sociales. Dans l'intéressante présentation de l'auteur, l'Union soviétique elle-même fait figure de « société coloniale » fondée sur 
une différenciation délibérément maintenue entre centre et périphérie. Et l'adoption contemporaine, dans les États d'Asie centrale, au Kirghizstan en particulier, de normes et de catégories russes comme étalons de développement social et de fierté nationale est ici vue comme conséquence de cette logique coloniale des sciences sociales en Asie centrale depuis le développement de ces dernières dans cette région au cours des années 1920-1930. C'est dire si l'histoire qui est contée dans ce livre, et qui englobe tout le cours $\mathrm{du} \mathrm{xx}^{\mathrm{e}}$ siècle, soulève des questions d'ordre général sur la relation entre savoir en sciences sociales et l'État, auxquelles s'ajoute aujourd'hui le rôle du «marché » dans ce que l'auteur appelle le monde postsocialiste. Quantité de questions classiques en sociologie de la recherche sont aussi invoquées au cours de l'ouvrage, à commencer par le caractère universel ou culturellement et historiquement marqué de la « science » et de la « vérité ».

3 Le premier chapitre détache les récits de la « transition " postsoviétique de ses postulats politiques et épistémologiques. Le deuxième propose une histoire sociale des sciences sociales en Asie centrale avant les indépendances, dans un cadre qui est celui des histoires du colonialisme, avec un intérêt particulier pour la problématique orientalisme/occidentalisme. Le troisième révise les récits soviétiques d'histoire des sciences sociales en Asie centrale du point de vue de leur présentation des relations entre ces disciplines et la pratique politique en URSS. Dans le suivant, l'auteur examine l'articulation de cet ethos scientifique et politique avec les questions "nationales" telles que ces dernières ont été traitées pendant la perestroïka - la demande d'autonomie des sociologues kirghizes s'associant avec la pratique d'une idéologie marxiste un tantinet plus critique. Dans le cinquième, consacré à la période de l'indépendance, l'auteur s'emploie à démontrer l'éviction des concepts marxistes auparavant dominants et l'adoption systématique de "programmes capitalistes jusqu'alors exclus », avec le maintien parallèle de l'éthique soviétique de la recherche postulant la nécessité d'un lien fort entre vérité scientifique et pratique politique de l'État. Le chapitre VI se veut l'illustration de l'impact de l'économie de marché et du changement politique depuis 1991 sur les pratiques professionnelles des sociologues du Kirghizstan - l'une des nouveautés observées étant une variété nouvelle de ces pratiques, conditionnée notamment par la situation désormais très diversifiée des institutions scientifiques du pays. Le septième et dernier chapitre étudie l'émergence d'une nouvelle éthique de la recherche et insiste sur la dimension non seulement académique mais aussi sociopolitique de ce phénomène.

4 En conclusion, l'auteur insiste sur le fait que les sciences sociales en Asie centrale soviétique et actuelle paraissent formées non seulement par des structures externes de domination, mais aussi par l'action des chercheurs eux-mêmes, négociant les « frontières entre vérité et politique ", dans le but de créer une discipline qui soit à la fois scientifiquement valide (selon des logiques qui sont celles de la recherche) et politiquement normative (c'est-à-dire faisant sens avec la logique du pouvoir, quel que soit ce dernier). Amsler diagnostique que l'émancipation intellectuelle, que semblaient annoncer les réformes dans le domaine de la recherche et de l'éducation, connaît une érosion certaine du fait de la "colonisation" de ces dernières par les demandes en provenance d'un État toujours plus soucieux de nationaliser discours et pratiques, ainsi que de la concurrence plus aiguë que jamais instaurée au sein de la communauté scientifique par l'instauration de mécanismes de marché. Bien que non dénué d'une dialectique assez simpliste, politiquement convenable et ignorant entre autres les processus de concurrence - souvent mortels - entre institutions de recherche instaurés 
par le stalinisme ou le brejnévisme (deux moments historiques perçus comme autant de blocs indivis), et bien qu'abusant d'usages non critiques d'un certain nombre de notions historiques comme celle de colonialisme, l'ouvrage propose une mise en relation tout à fait pionnière de l'URSS et du monde colonial occidental du point de vue de l'histoire des pratiques et des discours cognitifs ainsi que des politiques de recherche. En dépit du goût certain de l'auteur pour l'abstraction et des très nombreuses obscurités de son texte, la lecture de l'ouvrage doit être recommandée à toutes celles et ceux qu'intéressent à un titre ou à un autre l'histoire comparée et l'épistémologie des sciences humaines et sociales dans le domaine soviétique, de l'orientalisme à l'anthropologie. Même si, en arrêtant son récit à l'aube du Xxie siècle, l'auteur s'est privée de la possibilité d'enrichir sa réflexion par les changements brutaux de 2005 au Kirghizstan (ceux de cette année étant intervenus à une date très postérieure à la publication de son ouvrage).

\section{NOTES}

82. Voir en particulier T. Gieryn, «Boundary work and the demarcation of science from non-science: strains and interests in professional ideologies of scientists ", American Sociological Review, 48, 1983, p. 781-795. 